\title{
Validação de método para determinação de 3,4- metilenodioximetanfetamina (MDMA) em comprimidos de ecstasy por cromatografia em fase gasosa
}

\author{
Silvio Fernandes Lapachinske, Mauricio Yonamine*, Regina Lucia de Moraes Moreau \\ Laboratório de Análises Toxicológicas, Departamento de Análises Clínicas e Toxicológicas, Faculdade de Ciências \\ Farmacêuticas, Universidade de São Paulo
}

*Correspondência

M. Yonamine

Laboratório de Análises Toxicológicas Faculdade de Ciências Farmacêuticas - USP

Av. Prof. Lineu Prestes, 580 B13B 05508-900 -São Paulo - Brasil

E-mail: yonamine@usp.br
$O$ ecstasy é comercializado, de maneira ilegal, normalmente sob a forma de comprimidos, com cores, aspectos, dimensões e logotipos variados. Quimicamente, é a metilenodioximetanfetamina (MDMA), um composto sintético com propriedades estimulante central e alucinogênicas. Devido à grande expansão do abuso de ecstasy, também tem aumentado o número de casos de intoxicações, decorrentes diretamente da droga (MDMA e análogas) elou de eventuais adulterantes. Algumas substâncias análogas à MDMA, já identificadas em comprimidos de ecstasy são: metilenodioxietilanfetamina (MDEA), metilenodioxianfetamina (MDA), metanfetamina e anfetamina. Como possiveis adulterantes, geralmente são encontradas cafeina e efedrinas. O objetivo deste trabalho foi a validação de um método analítico para quantificar a MDMA em comprimidos ou cápsulas de ecstasy, através da cromatografia em fase gasosa com detector de nitrogênio/fósforo (GC/NPD). Além disso, substâncias análogas à MDMA e adulterantes também foram identificados. $O$ método, que consiste na dissolução direta da amostra em metanol, centrifugação e diluição do sobrenadante, demonstrou ser simples, rápido e eficiente. Os limites de detecção e quantificação para a MDMA foram respectivamente de 1,5 e 3,0 mg/100 mg de comprimido. Amostras de comprimidos e cápsulas apreendidos como sendo ecstasy provenientes de 25 lotes foram analisadas, apresentando considerável variabilidade na composição e na quantidade de MDMA.
Unitermos

- MDMA

- Comprimidos de ecstasy

- Cromatografia em fase gasosa

\section{INTRODUÇÃO}

A 3,4-metilenodioximetanfetamina (MDMA), derivada sintética da anfetamina, foi patenteada pela empre- sa farmacêutica alemã Merck, em 1914, com o intuito de ser um novo moderador do apetite. Entretanto, em função de sua baixa utilidade clínica, foi praticamente ignorada pela comunidade científica até a década de 1970, quando 
se relatou que produzia "um estado controlável de alteração da consciência com harmonia sensual e emocional", sugerindo que poderia ser usada como um adjuvante na psicoterapia (Downing, 1986; Ferigolo et al., 1998; Greer, Strassman, 1985; Greer, Tolbert, 1986).

No início da década de 1980, porém, a MDMA tornou-se popular como droga de uso recreacional, sendo conhecida também pelos nomes de ecstasy, "XTC", "E", "Adam", "MDM" e pílula do amor. Seu uso produz elevação da auto-estima com sensação de proximidade e intimidade com as pessoas ao redor. A comunicação e a relação com as pessoas melhoram e produz-se um sentimento de euforia com aumento da energia emocional e física (Dowling, 1986; Ferigolo et al., 1998; Greer, Tolbert, 1986; Nichols, 1986). Em doses excessivas, também pode produzir efeitos alucinogênicos. Mortes em decorrência do abuso de ecstasy são atribuídas a distúrbios cardiorrespiratórios, hipertermia e desidratação.

Nos últimos anos, aumento considerável do consumo de ecstasy tem sido observado mundialmente. $\mathrm{O}$ United Nations Office for Drug Control and Crime Prevention (UNODCCP) estima que aproximadamente 4,5 milhões de pessoas (a maioria adolescentes e adultos jovens) tenham utilizado ecstasy durante a década de 1990 (United Nations Office for Drug Control and Drug Prevention, 2000). Em 2000, de acordo com a Europol, 17,4 milhões de comprimidos de ecstasy foram apreendidos nos países membros da União Européia, correspondendo a aumento de quase $50 \%$, comparado com o ano de 1999 (Gimeno et al., 2003).

No Brasil, crescentes quantidades de ecstasy também têm sido apreendidas, culminando com a derrubada do primeiro laboratório de síntese de MDMA em São Paulo, no ano de 2000 (Baptista et al. 2002).

O ecstasy é consumido por via oral em comprimidos de várias formas, cores e tamanhos, geralmente contendo um logotipo que identifica o produto. Cápsulas gelatinosas são menos freqüentes, mas também têm sido encontradas. Essas preparações, contêm cerca de 50-150 mg de MDMA, sendo que algumas podem conter outros adulterantes como cafeína, efedrinas, paracetamol, cetamina e compostos análogos como a anfetamina, a metanfetamina, a 3,4-metilenodioxianfetamina (MDA) e a 3,4-metilenodioxietilanfetamina (MDEA) (Ferigolo et al., 1998; Siegel, 1986; Wolff et al., 1995).

Desta forma, a finalidade do presente trabalho foi o desenvolvimento de um método analítico para quantificar a MDMA em comprimidos ou cápsulas de ecstasy, utilizando a técnica de cromatografia em fase gasosa com detector de nitrogênio/fósforo (GC/NPD). Compostos análogos e adulterantes também puderam ser identifica- dos. Os resultados das análises de amostras de 25 diferentes lotes de apreensões realizadas em São Paulo (SP) também estão apresentados.

\section{MATERIAL E MÉTODOS}

\section{Soluções-padrão}

Soluções-padrão de anfetamina, metanfetamina, 3,4-metilenodioximetanfetamina (MDMA), 3,4metilenodioxianfetamina (MDA), 3,4-metilenodioxietilanfetamina (MDEA), efedrina, pseudoefedrina e norefedrina, na concentração de $1 \mathrm{mg} / \mathrm{mL}$ em metanol, foram obtidas da Radian International (Austin, EUA). Difenilamina foi obtida da Fisher Scientific (Pittsburg, EUA). Cafeína foi obtida da United States Pharmacopeia (Rockville, EUA).

\section{Amostras de comprimidos e cápsulas de ecstasy}

Amostras provenientes de 25 diferentes lotes apreendidos como sendo ecstasy, no período de 1996 a 2001, foram cedidas pelo Departamento de Investigações sobre Narcóticos (DENARC) e pelos extintos Serviço Técnico de Toxicologia Forense e Núcleo de Toxicologia Forense do Instituto Médico Legal de São Paulo e encaminhadas para o Laboratório de Análises Toxicológicas da Faculdade de Ciências Farmacêuticas da Universidade de São Paulo. Uma amostra de comprimido (REF 1) e uma amostra de cápsula (REF 2), cujas concentrações de 3,4metilenodioximetanfetamina (MDMA) foram determinadas pelo método, foram utilizadas nos ensaios de limite de detecção, limite de quantificação e precisão intra e interensaio.

\section{Preparo das amostras}

Cada comprimido foi triturado, homogeneizado e uma alíquota de $5 \mathrm{mg}$ foi dissolvida em $5 \mathrm{~mL}$ de metanol em um tubo de centrífuga de $15 \mathrm{~mL}$. No caso da amostra ser uma cápsula, o invólucro gelatinoso foi desprezado e uma alíquota de $5 \mathrm{mg}$ de seu conteúdo foi utilizada. Em seguida, o tubo foi submetido a banho de ultra-som por 10 minutos e centrifugado a $300 \mathrm{~g}$ por 5 minutos. Do sobrenadante, $50 \mathrm{~mL}$ foram transferidos para um balão de $1 \mathrm{~mL}$ juntamente com $50 \mathrm{~mL}$ de solução-padrão de difenilamina (padrão interno) na concentração de $100 \mathrm{mg} /$ $\mathrm{mL}$ e o volume completado com metanol. Da solução final, $1 \mathrm{~mL}$ foi injetado no equipamento de cromatografia em fase gasosa. 


\section{Cromatografia em fase gasosa}

Foi utilizado equipamento de cromatografia em fase gasosa Hewlett-Packard 6890 equipado com detector de nitrogênio/fósforo (GC/NPD) nas seguintes condições: injeção split (1:40); coluna capilar de sílica fundida 5\% fenilmetilsilicone com as dimensões de $25 \mathrm{~m} \mathrm{x} \mathrm{0,2} \mathrm{mm}$ x $0,33 \mu \mathrm{m}$ (HP-Ultra 2); temperatura do injetor, $270{ }^{\circ} \mathrm{C}$; gás de arraste, hélio a uma vazão de $0,6 \mathrm{~mL} / \mathrm{min}$; programação da temperatura no forno: $148^{\circ} \mathrm{C}(1 \mathrm{~min}), 10^{\circ} \mathrm{C} / \mathrm{min}$ até $200^{\circ} \mathrm{C}, 20^{\circ} \mathrm{C} / \mathrm{min}$ até $270^{\circ} \mathrm{C}(8 \mathrm{~min})$.

\section{Validação do método}

A validação do método foi realizada pelo estabelecimento de valores de limite de detecção, limite de quantificação, linearidade, precisão intra e interensaio e especificidade para a MDMA, conforme descrito abaixo:

\section{Limite de detecção e limite de quantificação}

O limite de detecção e o limite de quantificação foram obtidos pelo método empírico, que consistiu em analisar série de alíquotas do comprimido de ecstasy (REF 1) triturado e homogeneizado, diluídas em quantidades crescentes de excipientes (lactose $48 \%$, celulose microcristalina $48 \%$ e estearato de magnésio 4\%). O limite de detecção foi a menor concentração de MDMA obtida a partir da série de diluições que apresentou coeficiente de variação $(\mathrm{CV})$, que não excedeu $20 \%$ em seis replicatas. O limite de quantificação foi a menor concentração de MDMA que apresentou um coeficiente de variação $(\mathrm{CV})$ que não excedeu 10\% em seis replicatas (Chasin et al., 1998; Armbruster et al., 1994; Needleman, Romberg, 1990).

\section{Linearidade}

O estudo de linearidade foi conduzido pela injeção de 6 concentrações da solução-padrão de MDMA em triplicata, juntamente com o padrão interno difenilamina. As seguintes concentrações foram utilizadas: 1,$5 ; 10 ; 20$; $30 ; 40$ e $50 \mathrm{mg} / \mathrm{mL}$ em metanol, correspondentes às seguintes concentrações equivalentes nos comprimidos 3,0 ; 20; 40; 60; 80 e $100 \mathrm{mg}$ de MDMA por $100 \mathrm{mg}$ de comprimido ou cápsula ( $3 ; 20 ; 40 ; 60 ; 80$ e 100\% de MDMA).

\section{Precisão intra e interensaio}

A precisão intra e interensaio foi realizada analisando-se alíquotas das amostras de comprimido (REF 1) e da cápsula (REF 2), conduzidas em sextuplicata em três dias diferentes. Os valores foram expressos pelo coeficiente de variação do método $(\mathrm{CV})$.

\section{Especificidade}

A especificidade do método foi verificada utilizando compostos análogos e fármacos que poderiam estar presentes como adulterantes: anfetamina, metanfetamina, 3,4-metilenodioxianfetamina, 3,4-metilenodioxietilanfetamina, efedrina, pseudoefedrina, norefedrina e cafeína. As estruturas químicas dessas substâncias são apresentadas na Figura 1. Todas essas substâncias foram injetadas no equipamento de cromatografia em fase gasosa em solução metanólica a $10 \mu \mathrm{g} / \mathrm{mL}$.

\section{RESULTADOS}

O comprimido de ecstasy (REF 1) e a cápsula (REF 2) apresentaram concentração de MDMA, respectivamente, de $32,4 \%$ e $79,7 \%$, quando analisados pelo método proposto. Uma diluição de uma alíquota desse comprimido (REF 1) com excipientes na proporção de $1: 20$ foi necessária para se obter o limite de detecção do método, ou seja, uma concentração de 1,5 mg/100 mg (1,5\% de MDMA). O limite de quantificação foi obtido com a diluição do comprimido (REF 1) com excipientes na proporção de 1:10, gerando uma concentração de $3 \mathrm{mg} / 100 \mathrm{mg}$ ( $3 \%$ de MDMA).

A curva de calibração foi construída com concentrações de MDMA na faixa de 3 a 100\%. A equação da reta obtida foi: $y=0,126 x-0,40$, gerando um coeficiente de correlação $\left(\mathrm{r}^{2}\right)$ de 0,996 . Com relação ao estudo de especificidade, os resultados dos tempos de retenção absolutos e relativos à difenilamina (padrão interno) são apresentados na Tabela I.

A precisão intra e interensaio, expressa pelo coeficiente de variação (CV) do método, verificada para a análise do comprimido de ecstasy (REF 1) foi respectivamente de 5,4 e $0,8 \%$. A precisão intra e interensaio, verificada para a análise da cápsula de ecstasy (REF 2), foi respectivamente de 4,0 e $2,2 \%$.

Amostras de ecstasy ( 3 cápsulas e 22 comprimidos) provenientes de 25 lotes de apreensão realizados em São Paulo foram submetidos ao método para caracterização da sua composição ativa e determinação da concentração de MDMA presente em sua formulação. As amostras foram pesadas e identificadas conforme sua forma, cor e logotipo. O conteúdo de MDMA variou de 30,9 a 92,7 mg. Algumas amostras que possuíam o mesmo logotipo estampado, porém oriundas de lotes diferentes, apresentaram diferenças consideráveis na quantidade de MDMA. Isto sugere que não são provenientes do mesmo fabricante 
<smiles>CC(Cc1ccccc1)NC1CC2CCC21</smiles><smiles>CNC(C)Cc1ccc2c(c1)OCC2</smiles><smiles>C1CC2CC1C2</smiles><smiles>CCNC(C)Cc1ccc2c(c1)OCO2</smiles><smiles>C1=CC=C1</smiles><smiles>Cn1c(=O)c2c(ncn2C)n(C)c1=O</smiles>

B<smiles>CNC(C)Cc1ccccc1</smiles>

D<smiles>CNC(C)Cc1ccc2c(c1)OCO2</smiles>

\section{F}<smiles>CC(N)C(O)c1ccccc1</smiles>
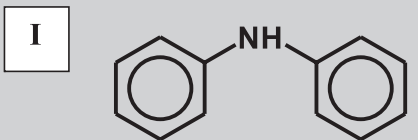

FIGURA 1 - Estruturas químicas das substâncias pesquisadas. (A) anfetamina, (B) metanfetamina, (C) 3,4metilenodioxianfetamina, (D) 3,4-metilenodioximetanfetamina, (E) 3,4-metilenodioxietilanfetamina, (F) norefedrina, (G) cafeína, (H) efedrina e (I) difenilamina.

TABELA I - Tempos de retenção absolutos e relativos à difenilamina (padrão interno) obtidos para as substâncias pesquisadas no estudo de especificidade do método

\begin{tabular}{lcc}
\hline Substância & $\begin{array}{c}\text { Tempo de retenção } \\
\text { absoluto (min) }\end{array}$ & $\begin{array}{c}\text { Tempo de retenção } \\
\text { relativo à difenilamina }\end{array}$ \\
\hline Anfetamina & 3,21 & 0,415 \\
Metanfetamina & 3,57 & 0,461 \\
3,4-Metilenodioxianfetamina (MDA) & 6,31 & 0,815 \\
3,4-Metilenodioximetanfetamina (MDMA) & 6,85 & 0,885 \\
3,4-Metilenodioxietilanfetamina (MDEA) & 7,30 & 0,943 \\
Difenilamina (padrão interno) & 7,74 & - \\
Efedrina & 5,20 & 0,671 \\
Pseudoefedrina & 5,19 & 0,671 \\
Norefedrina & 4,77 & 0,616 \\
Cafeína & 9,68 & 1,25 \\
\hline
\end{tabular}

ou que durante o processo de mistura entre o princípio ativo e os excipientes não ocorreu homogeneização eficiente. Em algumas amostras, a MDMA não foi encontrada, sendo substituída por substâncias análogas como a anfetamina, a metanfetamina e a MDEA. Os resultados estão apresentados na Tabela II. Um cromatograma da análise de um comprimido contendo MDMA e cafeína é apresentado na Figura 2. As fotos de alguns comprimidos e cápsulas analisados através do método proposto estão na Figura 3. 
TABELA II - Resultados obtidos com a análise de amostras apreendidas como sendo ecstasy, utilizando o método validado por cromatografia em fase gasosa

\begin{tabular}{|c|c|c|c|c|c|c|c|}
\hline Amostra & Forma & Cor & Logotipo & $\begin{array}{l}\text { Peso } \\
\text { (mg) }\end{array}$ & $\begin{array}{c}\text { Substâncias } \\
\text { ativas } \\
\text { encontradas }\end{array}$ & $\begin{array}{c}\text { Conc. de } \\
\text { MDMA } \\
(\%)\end{array}$ & $\begin{array}{c}\text { Qtde de } \\
\text { MDMA na } \\
\text { amostra (mg) }\end{array}$ \\
\hline 1 & $\begin{array}{l}\text { Comprimido } \\
\text { redondo }\end{array}$ & Bege & "TT" & 299,6 & MDMA & 23,3 & 69,8 \\
\hline 2 & $\begin{array}{l}\text { Comprimido } \\
\text { redondo }\end{array}$ & Bege & "TT" & 307,3 & MDMA & 15,9 & 48,9 \\
\hline 3 & $\begin{array}{l}\text { Comprimido } \\
\text { redondo }\end{array}$ & Bege & "Pac man" & 288,7 & MDMA & 24,5 & 70,7 \\
\hline 4 & $\begin{array}{l}\text { Comprimido } \\
\text { redondo }\end{array}$ & Bege & "Mitsubishi" & 312,3 & MDMA & 23,7 & 74,0 \\
\hline 5 & $\begin{array}{l}\text { Comprimido } \\
\text { redondo }\end{array}$ & Bege & "Cavalo Ferrari" & 272,5 & MDMA & 20,7 & 56,4 \\
\hline 6 & $\begin{array}{l}\text { Comprimido } \\
\text { redondo }\end{array}$ & Bege & "Cavalo alado" & 249,6 & MDMA & 17,8 & 44,4 \\
\hline 7 & $\begin{array}{l}\text { Comprimido } \\
\text { redondo }\end{array}$ & Bege & "Euro" & 343,0 & MDMA & 19,9 & 68,3 \\
\hline 8 & $\begin{array}{l}\text { Comprimido } \\
\text { redondo }\end{array}$ & Bege & "Mitsubishi" & 333,0 & MDMA & 24,8 & 82,6 \\
\hline 9 & $\begin{array}{l}\text { Comprimido } \\
\text { redondo }\end{array}$ & Bege & "Cavalo Ferrari" & 277,4 & MDMA & 19,3 & 53,5 \\
\hline 10 & $\begin{array}{l}\text { Comprimido } \\
\text { redondo }\end{array}$ & Bege & "TT" & 295,6 & MDMA & 19,4 & 57,3 \\
\hline 11 & $\begin{array}{l}\text { Comprimido } \\
\text { redondo }\end{array}$ & Bege & "Cavalo Ferrari" & 271,2 & MDMA & 32,7 & 88,7 \\
\hline 12 & Cápsula & Bege & - & 116,3 & MDMA & 79,7 & 92,7 \\
\hline 13 & $\begin{array}{l}\text { Comprimido } \\
\text { redondo }\end{array}$ & Bege & "Flor" & 279,7 & MDMA & 22,2 & 62,1 \\
\hline 14 & $\begin{array}{l}\text { Comprimido } \\
\text { redondo }\end{array}$ & Bege & “Tulipa" & 235,4 & MDMA & 30,1 & 70,8 \\
\hline 15 & Cápsula & Bege & - & 82,3 & MDMA & 61,2 & 50,4 \\
\hline 16 & $\begin{array}{l}\text { Comprimido } \\
\text { oval }\end{array}$ & Bege & "Fogueira" & 482,1 & MDMA & 6,4 & 30,9 \\
\hline 17 & $\begin{array}{l}\text { Comprimido } \\
\text { redondo }\end{array}$ & Rosa pálido & “\$”(cifrão) & 258,4 & Anfetamina Cafeína & - & - \\
\hline 18 & Cápsula & Verde & - & 85,7 & Metanfetamina & - & - \\
\hline 19 & $\begin{array}{l}\text { Comprimido } \\
\text { redondo }\end{array}$ & Marrom & Liso & 214,1 & MDMA & 29,5 & 63,2 \\
\hline 20 & $\begin{array}{l}\text { Comprimido } \\
\text { redondo }\end{array}$ & Bege & Liso & 277,4 & MDMA & 20,4 & 56,6 \\
\hline 21 & $\begin{array}{l}\text { Comprimido } \\
\text { redondo }\end{array}$ & Ocre & Liso & 199,3 & MDMA & 24,6 & 49,1 \\
\hline 22 & $\begin{array}{l}\text { Comprimido } \\
\text { redondo }\end{array}$ & Bege & "Índio" & 301,4 & MDMA Cafeína & 19,9 & 60,0 \\
\hline 23 & $\begin{array}{l}\text { Comprimido } \\
\text { redondo }\end{array}$ & Cinza & Liso & 296,5 & MDEA & - & - \\
\hline 24 & $\begin{array}{l}\text { Comprimido } \\
\text { redondo }\end{array}$ & Rosa & Liso & 303,6 & MDMA & 21,9 & 66,5 \\
\hline 25 & $\begin{array}{l}\text { Comprimido } \\
\text { redondo }\end{array}$ & Rosa & "Diamante" & 198,3 & MDMA & 30,6 & 60,7 \\
\hline
\end{tabular}

MDMA = 3,4-metilenodioximetanfetamina, MDEA = 3,4-metilenodioxietilanfetamina 


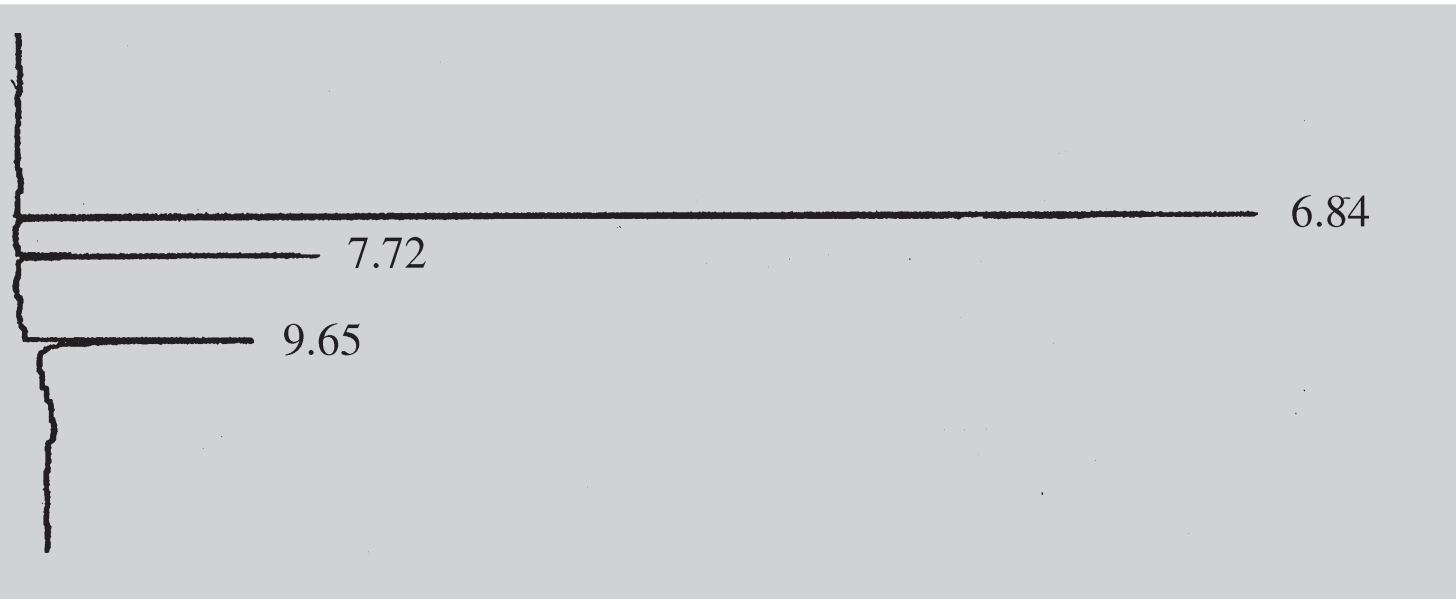

FIGURA 2 - Cromatograma obtido com a análise de uma amostra de comprimido de ecstasy identificado na TABELA II pelo número 22 ("Índio") contendo MDMA (TR=6,84) e cafeína (TR=9.65). Padrão interno= difenilamina (TR=7.72). Tempo de retenção (TR) em minutos.

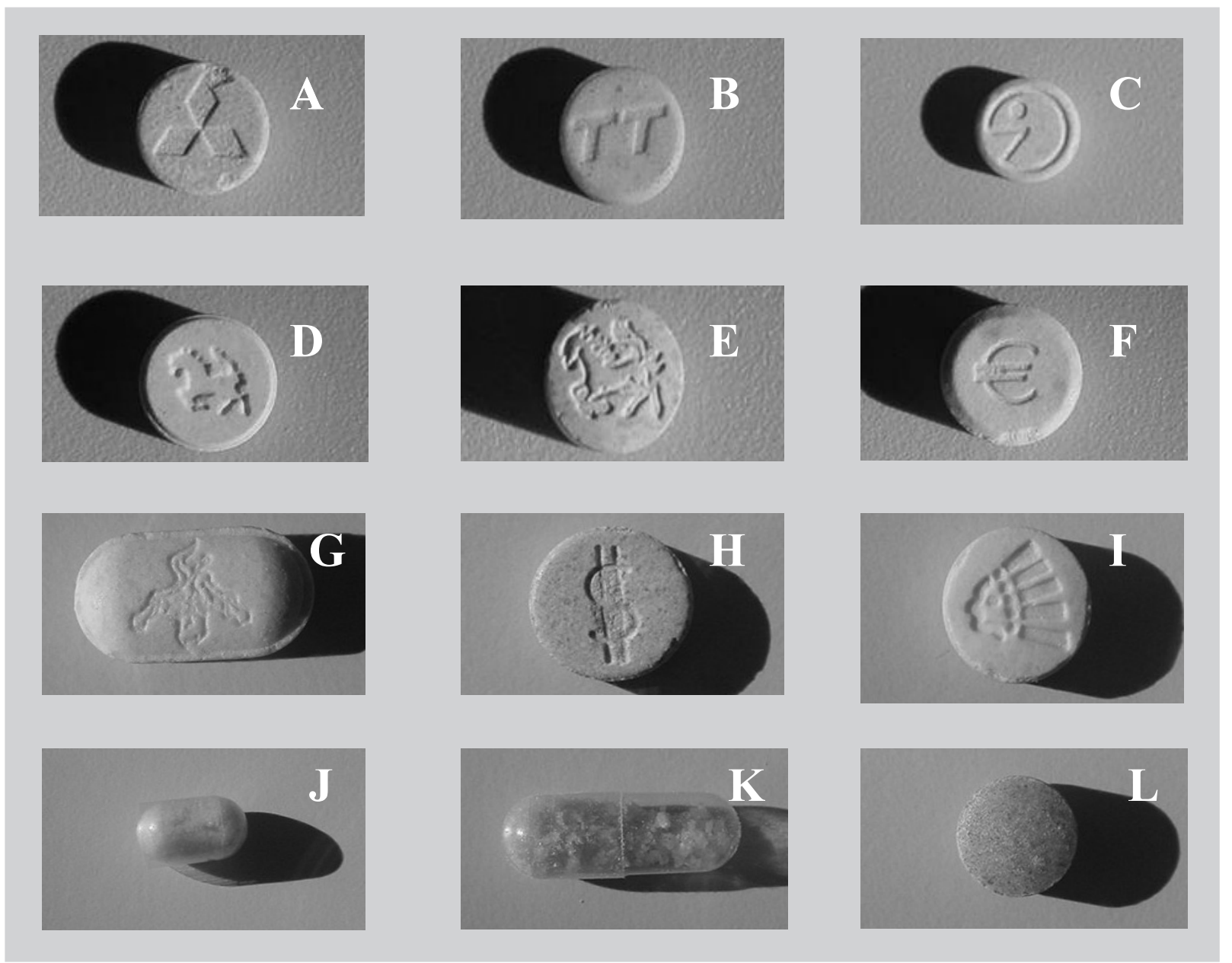

FIGURA 3 - Algumas amostras de comprimidos e cápsulas apreendidos como sendo ecstasy cuja composição foi determinada pelo método proposto por cromatografia em fase gasosa. (A) Comprimido "Mitsubishi", (B) "TT", (C) "Pac man”, (D) “Cavalo Ferrari”, (E) “Cavalo alado”, (F) “Euro”, (G) “Fogueira”, (H) “Cifrão”, (I) “Índio”, (J) Cápsula verde, (K) Cápsula bege e (L) Comprimido marrom liso. 


\section{DISCUSSÃO}

Alguns métodos para determinação da composição de amostras de ecstasy têm sido relatados na literatura científica, utilizando técnicas de espectroscopia no infravermelho (Sondermann, Kovar, 1999), eletroforese capilar (Piette, Parmentier, 2002), cromatografia líquida (Cole et al., 2002; Giroud et al., 1997; Sadeghipour, Veuthey, 1997), cromatografia em fase gasosa e espectrometria de massa associada à cromatografia em fase gasosa (Silva et al., 1998). Os resultados desses trabalhos têm demonstrado que não somente a MDMA está presente em amostras apreendidas como sendo ecstasy. Muitas vezes, compostos análogos, como a MDA e a MDEA podem ser as substâncias ativas encontradas na composição desses comprimidos e misturas com outros estimulantes como cafeína e efedrina não são incomuns. Também tem-se verificado que não somente a composição dos comprimidos de ecstasy têm apresentado variações, mas também a concentração e quantidade de MDMA encontrada. Estudo realizado por Sherlock et al. (1999) demonstrou que concentrações de MDMA variavam até 70 vezes entre os comprimidos analisados. Em outro estudo, realizado por Cole et al. (2002), comprimidos apreendidos na Inglaterra $(\mathrm{n}=136)$ apresentavam conteúdo de MDMA, que variava de 20 a $109 \mathrm{mg}$.

No presente trabalho, a cromatografia em fase gasosa com detector de nitrogênio/fósforo foi utilizada como técnica para a quantificação da MDMA presente em comprimidos apreendidos em São Paulo (SP) como sendo ecstasy. $\mathrm{O}$ método, baseado na dissolução direta de uma alíquota da amostra com metanol, demonstrou ser procedimento prático e rápido, em comparação com outros métodos em que são utilizadas fases de extração e pré-tratamento da amostra (Gimeno et al., 2003; Silva et al., 1998). Boa separação cromatográfica também foi obtida no estudo da especificidade, sendo possível detectar simultaneamente compostos análogos e adulterantes. A alíquota utilizada no método $(5 \mathrm{mg})$ demonstrou ser representativa da amostra total, pois os estudos de precisão intra e interensaio realizados com um comprimido e uma cápsula de ecstasy apresentaram baixos coeficientes de variação $(\mathrm{CV}<6 \%)$.

Amostras de ecstasy apreendidas em São Paulo (SP) foram submetidas ao método validado para identificação de sua composição ativa. Embora os comprimidos apresentassem uma variedade na coloração, sugerindo diferentes tipos de pigmentos na sua composição, isto em nada interferiu na análise cromatográfica, assim como os excipientes da formulação também não interferiram.
Apesar da pequena quantidade de comprimidos e cápsulas analisados, variabilidade considerável foi encontrada tanto na composição ativa (MDMA, compostos análogos e adulterantes) como na quantidade de MDMA presente nas amostras pesquisadas. Em algumas amostras, inclusive, a MDMA não foi encontrada, sendo substituída por substâncias com maior potencial de causar dependência como a anfetamina e a metanfetamina. Essa variabilidade pode ter sérias implicações pois usuários da droga podem estar expostos a diversas doses e/ou diversas combinações com outros tipos de agentes psicoativos. Levando-se em consideração que usuários de ecstasy podem ser consumidores de outras drogas como cocaína, maconha, bebidas alcoólicas e alucinógenos (Baptista et al., 2002), o risco de intoxicações se torna ainda mais elevado.

É possível que usuários de ecstasy aumentem a ingestão do número de comprimidos por vez, para obter o efeito desejado caso a concentração de MDMA esteja baixa na droga normalmente utilizada. Se houver mudança no fornecedor, para comprimidos mais concentrados em MDMA e se mantiver o mesmo padrão de uso, uma superdosagem pode ocorrer, aumentando o risco de intoxicações.

Portanto, a identificação dos componentes ativos do ecstasy se torna necessária, fornecendo informações adicionais para os profissionais da área emergencial e de tratamento, envolvidos na elucidação de casos de abuso da droga e intoxicações. Além disso, o esclarecimento das características das misturas de ecstasy no Brasil, através de análises toxicológicas sistemáticas, poderia dar indícios de possíveis fontes de produção, distribuição e origem da droga.

\section{CONCLUSÕES}

O método desenvolvido e validado para determinação de MDMA por cromatografia em fase gasosa com detector de nitrogênio/fósforo demonstrou ser simples, rápido e eficiente, podendo ser aplicado em laboratórios de toxicologia que dispõem deste equipamento. Nas amostras de ecstasy apreendidas em São Paulo (SP), variabilidade considerável foi encontrada tanto na composição ativa (MDMA, compostos análogos e adulterantes) como na quantidade de MDMA. Como não existe controle de qualidade no mercado de drogas ilícitas, observa-se risco adicional de intoxicação devido à possibilidade de exposição a concentrações diversas e combinação com outros fármacos psicoativos presentes nos comprimidos $\mathrm{e}$ cápsulas de ecstasy. 


\section{ABSTRACT}

\section{Validation of a gas-chromatographic method for the determination of 3,4-methylenedioxymethamphetamine (MDMA) in ecstasy tablets}

Ecstasy is illegally commercialized in the form of tablets with different aspects, colors, sizes, and logotypes. Chemically, ecstasy is 3,4-methylenedioxymethamphetamine (MDMA), a synthetic compound with stimulant and hallucinogenic proprieties. Due to the great expansion of ecstasy abuse, the number of cases of intoxications by MDMA, analogs and eventual adulterant compounds has also increased. Some MDMA analog substances, such as 3,4-methylenedioxyethylamphetamine (MDEA), 3,4-methylenedioxyamphetamine (MDA), methamphetamine and amphetamine have already been identified in ecstasy tablets. Caffeine and ephedrines are the most common adulterants also found. The aim of this paper is to describe the validation of an analytical method to quantify MDMA in ecstasy tablets and capsules. Gaschromatography with nitrogen/phosphorous detector was used in the method, which consisted in the direct dissolution of the sample in methanol, centrifugation and convenient dilution of the supernatant. Analog substances to MDMA and adulterants were also identified. The limits of detection and quantification (LOQ and LOD) for MDMA were 1.5 and $3.0 \mathrm{mg} / 100 \mathrm{mg}$ of tablet. Samples from 25 lots of tablets seized in the city of São Paulo were analyzed showing a considerable variability in composition and quantity of MDMA.

UNITERMS: MDMA. Ecstasy tablet. Gaschromatography

\section{REFERÊNCIAS BIBLIOGRÁFICAS}

ARMBRUSTER, D. A., TILLMAN, M. D., HUBBS, L. M. Limit of detection (LOD)/ Limit of quantitation (LOQ): comparison of the empirical and the statistical methods exemplified with GC-MS assays of abused drugs. Clin. Chem., Washington, v.40, n.7, p.1233-1238, 1994.

BAPTISTA, M. C., NOTO, A. R., NAPPO, S., CARLINI, E. A. O uso de êxtase (MDMA) na cidade de São Paulo e imediações: um estudo etnográfico. J. Bras. Psiquiatria, Rio de Janeiro, v.51, n.2, p.81-89, 2002.
CHASIN, A. A. M., NASCIMENTO, E. S., RIBEIRONETO, L. M., SIQUEIRA, M. E. P. B., ANDRAUS, M. H., SALVADORI, M. C., FERNÍCOLA, N. A. G., GORNI, R., SALCEDO, S. Validação de métodos em análises toxicológicas: uma abordagem geral. Rev. Bras. Toxicol., São Paulo, v.11, n.1, p.1-6, 1998.

COLE, J. C., BAILEY, M., SUMNALL, H. R., WAGSTAFF, G. F., KING, L. A. The content of ecstasy tablets: implications for the study of their long-term effects. Addiction, Oxford, v.97, p.1531-1536, 2002.

DOWNING, J. The psychological and physiological effects of MDMA on normal volunteers. J. Psychoactive Drugs, San Francisco, v.18, p.335-340, 1986.

FERIGOLO, M., MEDEIROS, F. B., BARROS, H. M. T. "Êxtase": revisão farmacológica. Rev. Saúde Pública, São Paulo, v.32, n.5, p.487-495, 1998.

GIMENO, P., BESACIER, F., CHAUDRON-THOZET, H. Optimization of extraction parameters for the chemical profiling of 3,4-methylenedioximethamphetamine (MDMA) tablets. Forensic Sci. Int., Lausanne, v.132, p.182-194, 2003.

GIROUD, C., AUGSBURGER, M., SADEGHIPOUR, F., VARESIO, E., VEUTHEY, J. L, RIVIER, L. Ecstasy-la situation en Suisse romande. Praxis, Bern, v.86, p.510$523,1997$.

GREER, G., STRASSMAN, R. Information on ecstasy. Am. J. Psychiatry, Arlington, v.142, p.1391, 1985.

GREER, G., TOLBERT, R. Subjective reports of the effects of MDMA in a clinical setting. J. Psychoactive Drugs, San Francisco, v.18, p.318-327, 1986.

UNITED NATIONS OFFICE FOR DRUG CONTROL AND CRIME PREVENTION. World Drug Report 2000. New York: United Nations, 2000. 173p.

NEEDLEMAN, S. B., ROMBERG, R. W. Limits of linearity and detection for some drugs of abuse. J. Anal. Toxicol., Niles, v.14, p.34-38, 1990.

NICHOLS, D. E. Differences between the mechanism of action of MDMA, MBDB and classic hallucinogens. Identification of a new therapeutic class: entactogens. $J$. Psychoactive Drugs, San Francisco, v.18, p.305-313, 1986. 
PIETTE, V., PARMENTIER, F. Analysis of illicit amphetamine seizures by capillary zone eletrophoresis. J. Chromatogr. A, Amsterdam, v.979, p.345-352, 2002.

SADEGHIPOUR, F., VEUTHEY, J. L. Sensitive and selective determination of methylenedioxylated amphetamines by high-performance liquid chromatography with fluorimetric detection. $J$. Chromatogr. A., Amsterdam, v.787, p.137-143, 1997.

SHERLOCK, K., WOLFF, K., HAY, A. W., CONNER, M. Analysis of illicit ecstasy tablets: implications for clinical management in the accident and emergency department. J. Accid. Emerg. Med., London, v.16, n.3, p.194-197, 1999.

SIEGEL, R. K. MDMA nonmedical use and intoxication. $J$. Psychoactive Drugs., San Francisco, v.18, p.349-354, 1986.
SILVA O. A., YONAMINE, M., REINHARDT, V. E. Identificação de 3,4-metilenodioximetanfetamina (MDMA) e compostos relacionados por cromatografia em fase gasosa e espectrometria de massa em comprimidos de ecstasy apreendidos em São Paulo. Rev. Farm. Bioquím. Univ. São Paulo, São Paulo, v.34, n.1, p.33-37, 1998.

SONDERMANN, N., KOVAR, K. A. Screening experiments of ecstasy street samples using near infrared spectroscopy. Forensic Sci. Int., Lausanne, v.106, p.147156, 1999.

WOLFF, K., HAY, A., SHERLOCK, K., CONNER, M. Contents of "ecstasy". Lancet, London, v.346, p.11001101, 1995.

Recebido para publicação em 08 de julho de 2003. 